\title{
The progression of neurofeedback: an evolving paradigm in addiction treatment and relapse prevention
}

\begin{abstract}
Addiction is characterized by improper engagement of neurobiological systems involved in adaptive decision-making. The prevalence of relapse among addiction patients may be explained by the persistence of maladaptive patterns of synaptic connectivity. An effective approach to addiction treatment and relapse prevention may thus rely on enhancing the brain's capacity for neuroplasticity and self-regulation. A growing body of evidence indicates that neurofeedback is capable of promoting neuroplasticity, resolving maladaptive activity, and improving self-regulation.
\end{abstract}

Keywords: addiction, relapse, self-regulation, synaptic connectivity, neurofeedback, neuroplasticity
Volume 3 Issue 3 - 2017

\author{
Keerthy R Sunder, ${ }^{1,2}$ Jeffrey L Bohnen' \\ 'Mind \& Body Treatment and Research Institute, USA \\ ${ }^{2}$ UC Riverside School of Medicine, USA
}

Correspondence: Keerthy R Sunder, Medical Director, Mind \& Body Treatment and Research Institute, Riverside, CA, USA, Email DrKeerthy@mbtrins.com

Received: March 21, 2017| Published: April 20, 2017
Abbreviations: TOVA, test of variables of attention; MMPI, minnesota multiphasic personality inventory; cAMP, cyclic adenosine monophosphate; CREB, camp response element binding protein; MCI, mild cognitive impairment

\section{Introduction}

\section{Recognizing addiction as a public health crisis}

A recent report published by the Center for Behavioral Health Statistics and Quality found that approximately one in seven Americans is expected to develop a substance use disorder at some point in their lives. ${ }^{1}$ In 2015, 20.8 million people aged 12 or older in the United States had a substance abuse disorder, indicating that the number of Americans suffering from addiction is similar to the number of Americans suffering from diabetes. ${ }^{2}$ Moreover, the prevalence of substance use disorders is nearly 1.5 times that of all cancers combined. ${ }^{3}$

In 2014, over 47,000 people died from a drug overdose. This figure includes nearly 30,000 people who died from an overdose involving prescription drugs, which is more than in any previous year on record. Heroin overdoses have more than tripled between 2010 and $2014 .{ }^{4}$ Alcohol misuse, which contributes to 88,000 deaths per year in the United States, was found to be the nation's fourth leading preventable cause of death. ${ }^{5,6}$ Approximately one in ten deaths among working adults in the United States is caused by alcohol abuse. ${ }^{5}$ The health care expenses, lost productivity, and criminal justice costs related to substance abuse are estimated to cost the federal government $\$ 442$ billion dollars each year. ${ }^{7.8}$ In response to this public health crisis, the U.S. Surgeon General published a report declaring that addiction must be approached as a chronic illness, alongside conditions such as heart disease, diabetes, and cancer. ${ }^{9}$

\section{Current issues in addiction treatment and relapse pre- vention}

Addiction is characterized by improper engagement of the systems involved in adaptive decision-making. ${ }^{10}$ Deficiencies in executive functions such as working memory allow substance use behavior to be guided more strongly by automatic, impulsive processes. ${ }^{11}$ Genetic polymorphisms or stress may induce a hypodopaminergic trait/state, predisposing individuals to instinctively seek out substances or behaviors that stimulate dopaminergic activity. ${ }^{12,13}$ Substance use may thus engage a vicious cycle, as addictive drugs have been implicated to damage brain regions involved in higher-order functioning, further reducing self-regulatory capabilities. ${ }^{14}$

Although traditional methodologies such as counseling, group therapy, and medication have produced some success in treating addiction, relapse rates remain high. Bailey et al. reported that $90 \%$ of inpatient opioid detoxification patients relapsed within a year of treatment. ${ }^{15} \mathrm{~A}$ key factor accounting for the likelihood of relapse among addiction patients may be the persistence of aberrant neurobiological changes affecting reward prediction and motivation. It is plausible that traditional therapies may only address maladaptive patterns of synaptic connectivity to a limited extent, preserving some aberrant pathways. In times of stress or anxiety, addicts may abandon rational logic in favor of emotional impulse, activating their fight-orflight response and potentially prompting reengagement of aberrant neurobiological pathways, whose association with a sense of relief has been preserved. In a sample of cocaine addicts, stress-related increases in craving and associated hypothalamic-pituitary-adrenal axis responses were predictive of relapse outcomes. ${ }^{16}$ Although a "magic bullet" cure for addiction continues to evade researchers, an effective approach to addiction treatment and relapse prevention may rely on resolving core neurobiological issues affecting reward prediction, motivation, and adaptive decision-making.

\section{Synaptic changes underlying addiction}

Although the full diversity of drug effects involves a wide array of brain regions and mechanisms, most addictive drugs share the common property of promoting striatal dopamine release. ${ }^{17,18}$ Dopaminergic stimulation of gene expression is associated with long-lasting changes in synaptic networks, supporting the notion that dopamine may operate as a modulator of learning mechanisms. ${ }^{19}$ Specifically, dopamine has been implicated to play a key role in reward prediction and psychomotor activation. Fluctuations in 
dopamine cell firing rates strongly resemble "error signals" in models of reinforcement learning. ${ }^{10,20}$ Stimulation of dopamine neurons has been shown to modulate corticostriatal synaptic strength in direct correlation with behavioural reinforcement. ${ }^{21}$ Dopamine cells may thus play an integral role in a natural process designed to help shape behavior in accordance with reward prediction.

Following this logic, addictive drugs can be understood as "hackers" of this system, as they may reinforce maladaptive motivations and behaviors. Addictive drugs can engage molecular mechanisms normally involved in associative learning, including stimulation of dopamine D1 receptors, activation of the cAMP/PKA/ CREB signal transduction pathway, altered gene expression, and synaptic rearrangements. ${ }^{19}$ The persistence of drug addiction may reflect the persistence of altered patterns of synaptic connectivity created through these mechanisms. With an understanding of these mechanisms, it follows that an effective approach to addiction treatment and relapse prevention may need to address maladaptive patterns of synaptic connectivity and improve the brain's capacity for self-regulation.

\section{The emergence of neurofeedback as a recovery re- source}

Neurofeedback is an emerging treatment modality that involves the use of a brain-computer interface to display a patient's instantaneous brainwave activity on a monitor. By reflecting electrical activity across targeted brain regions, this procedure generates a "neural mirror," allowing the brain to observe its own activity.

Neurofeedback was implemented in the early 1970's, but it was not until the late 1980's that technological advancements optimized the treatment. During the late 1980's and early 1990's, Peniston \& Kulkosky ${ }^{22}$ developed an innovative neurofeedback protocol that was shown to increase alpha and theta brain wave production, normalize personality measures, prevent increases in beta-endorphin levels, and prolong prevention of relapse among alcoholic patients. ${ }^{22}$ Notably, the protocol achieved significant improvements in samples that included many subjects for whom pharmacological treatment had not proven beneficial.

In 2005, Scott et al. tested the effectiveness of a neurofeedback protocol on a mixed substance abusing population to assess whether or not the breakthroughs achieved with alcoholic patients could extend to abusers of other substances. ${ }^{23}$ In comparison to control subjects who underwent traditional therapy, experimental subjects who underwent neurofeedback training in conjunction with traditional therapy were nearly twice as likely to not drop out of treatment. The experimental group showed statistically significant improvement on five of the ten personality scales administered in the Minnesota Multiphasic Personality Inventory (MMPI). Experimental subjects also demonstrated statistically significant improvement on the Test of Variables of Attention (TOVA), which was not observed among control subjects. At the 12-month follow-up, $77 \%$ of the experimental subjects were abstinent, compared to $44 \%$ of the control subjects. Addictions for all drug types were successfully treated by the same neurofeedback protocol; there was no significant interaction between drug type used and abstinence rate among patients who underwent neurofeedback training. These findings suggest that neurofeedback can be used to target core neurobiological changes underlying the full spectrum of substance use disorders.
In an eight-year analysis (2006-2014) of 5,746 patients, Othmer $\mathrm{S} \&$ Othmer $\mathrm{SF}^{24}$ found that two-thirds of patients who initially scored poorly on measures of impulsivity (assessed via a Continuous Performance Test) attained normal functioning within twenty sessions of neurofeedback. ${ }^{24}$ Notably, many patients actually scored above functional norms once the therapy was complete. These results can be understood within the framework of an optimum functioning model. According to this model, the brain naturally processes self-relevant information to optimize its functioning. The enhanced cognitive performance observed among these patients may be explained by a mechanism in which the informational network is augmented, improving the brain's capacity to rewire itself in its innate quest for functional optimization.

Longitudinal follow-up data summarized by Othmer S \& Othmer SF showed that addiction patients who underwent neurofeedback training were three times more likely to be abstinent one year after treatment $(75 \%$ success rate among experimental subjects, in comparison to $25 \%$ among control subjects). ${ }^{25}$ A ten-year followup clinical evaluation of patients who had undergone the original Peniston \& Kulkosky protocol provided additional evidence for the long-term effectiveness of this intervention. ${ }^{22}$ These findings may be explained in part by fundamental shifts in regulatory dynamics, as neurofeedback targets right hemispheric brain regions involved in arousal regulation, affect regulation, and autonomic regulation. ${ }^{24}$

\section{Discussion}

Altered functional connectivity has been postulated to be a key failure mechanism in addiction psychopathology. ${ }^{26}$ However, by observing its own dysregulated state via neurofeedback, the brain can be propelled into novel state configurations, which are susceptible to reinforcement and consolidation. ${ }^{24}$ Ros et al. ${ }^{27}$ observed significant changes in neuronal activity following a session of neurofeedback training, which lasted for over 20 minutes. ${ }^{27}$ Addiction patients who underwent neurofeedback training reported experiencing dysphoria when they used a substance following the treatment, indicating that the intervention may have rewired the brain's response to substance use. ${ }^{23,28}$ Neurofeedback training may thus operate by promoting neuroplasticity, facilitating adjustments in neuronal activity that support optimum functioning. More specifically, neurofeedback has been postulated to activate astrocytic mechanisms that facilitate synaptogenesis and manage network excitability through capillary blood flow. ${ }^{29-37}$ Astrocytes function as the principal storage sites of glycogen granules in the central nervous system and may provide neurons with vital energetic substrates ${ }^{38-44}$ Astrocytes also operate as important mediators of neurotoxic events. ${ }^{45}$ By influencing astrocytic activity, neurofeedback may enhance the brain's ability to prune synaptic pathways, improving self-regulation.

A "brain fitness" program that featured neurofeedback reversed effects of mild cognitive impairment (MCI), achieving statistically significant improvements in cognitive function for $84 \%$ of patients. ${ }^{46}$ Among the sample of 17 patients who underwent a post-program quantitative MRI, 12 patients exhibited either no atrophy or an actual growth in hippocampal volume. These findings support the conclusion that brain training with neurofeedback can improve cognitive function and potentially reverse hippocampal atrophy in patients with MCI. Neurofeedback has also been shown to significantly improve executive functioning and working memory. ${ }^{47,48}$ By improving regulatory capabilities and executive functions, neurofeedback may 
provide a crucial buffer against relapse, which has been linked to impaired executive functions such as working memory. ${ }^{11,49}$ In addition, neurofeedback training may promote a more relaxed state and lead to better perceived control of stress, thereby addressing another key factor in relapse prevention. . $2,50^{2}$

\section{Conclusion}

Although further research is necessary to elucidate the precise mechanisms by which neurofeedback optimizes brain function, accumulating research shows promise for the application of this emerging technology in the field of addiction medicine.

\section{Acknowledgements}

None.

\section{Conflict of interest}

The author declares no conflict of interest.

\section{References}

1. Center for Behavioral Health Statistics and Quality. Results from the 2015 National Survey on Drug Use and Health: Detailed tables. Rockville, Substance Abuse and Mental Health Services Administration, Maryland, USA, 2016.

2. Centers for Disease Control and Prevention. National Diabetes Statistics Report: Estimates of Diabetes and Its Burden in the United States (2014) Atlanta, GA:US Department of Health and Human Services. 2014.

3. Howlader N \& Noone AM, Editors. SEER Cancer Statistics Review, 1975-2013. National Cancer Institute, Bethesda, USA, 2016.

4. Rudd RA, Aleshire N, Zibbel JE, et al. Increases in drug and opioid overdose deaths-United States, 2000-2014. MMWR Morb Mortal Wkly Rep. 2016;64(50-51):1378-1382.

5. Stahre M, Roeber J, Kanny D, et al. Contribution of excessive alcohol consumption to deaths and years of potential life lost in the United States. Prev Chronic Dis. 2014;11:(E109).

6. Mokdad AH, Marks JS, Stroup DF, et al. Actual causes of death in the United States, 2000. JAMA. 2004;291(10):1238-1245.

7. Sacks JJ, Gonzales KR, Bouchery EE, et al. 2010 national and state costs of excessive alcohol consumption. Am J Prev Med. 2015;49(5):e73-e79.

8. National Drug Intelligence Center. National drug threat assessment. Washington, US Department of Justice. 2011.

9. US Department of Health and Human Services Office of the Surgeon General (HHS). Facing Addiction in America: The Surgeon General's Report on Alcohol, Drugs, and Health. Washington, USA, 2016.

10. Berke JD. Learning and memory mechanisms involved in compulsive drug use and relapse. Methods Mol Med. 2003;79:75-101.

11. Houben K, Wiers RW, Jansen A. Getting a grip on drinking behaviour: training working memory to reduce alcohol abuse. Psychol Sci. 2011;22(7):968-975.

12. Blum K, Oscar Berman M, Demetrovics Z, et al. Genetic Addiction Risk Score (GARS): molecular neurogenetic evidence for predisposition to Reward Deficiency Syndrome (RDS). Mol Neurobiol. 2014;50(3):765796.

13. Comings DE, Blum K. Reward deficiency syndrome: genetic aspects of behavioural disorders. Prog Brain Res. 2000;126:325-341.

14. Crews FT, Boettiger CA. Impulsivity, Frontal Lobes and Risk for Addiction. Pharmacol Biochem Behav. 2009;93(3):237-247.
15. Bailey GL, Herman DS, Stein MD. Perceived Relapse Risk and Desire for Medication Assisted Treatment among Persons Seeking Inpatient Opiate Detoxification. J Subst Abuse Treat. 2013;45(3):302-305.

16. Sinha R, Garcia M, Paliwal P, et al. Stress-Induced Cocaine Craving and Hypothalamic-Pituitary-Adrenal Responses Are Predictive of Cocaine Relapse Outcomes. Arch Gen Psychiatry. 2006;63(3):324-331.

17. Di Chiara G, Imperato A. Drugs abused by humans preferentially increase synaptic dopamine concentrations in the mesolimbic system of freely moving rats. Proc Natl Acad Sci USA. 1988;85(14):5274-5278.

18. Kuczenski R, Segal DS, Aizenstein ML. Amphetamine, cocaine, and fencamfamine: relationship between locomotor and stereotypy response profiles and caudate and accumbens dopamine dynamics. $J$ Neurosci. 1991;11(9):2703-2712.

19. Berke JD, Hyman SE. Addiction, dopamine, and the molecular mechanisms of memory. Neuron. 2000;25(3):515-532.

20. Sutton RS, Barto AG. Reinforcement Learning: An Introduction. 2nd ed. The MIT Press, Cambridge, MA, UK, 1998. p. 1-334.

21. Reynolds JN, Hyland BI, Wickens JR. A cellular mechanism of rewardrelated learning. Nature. 2001;413:67-70.

22. Eugene O Peniston, Peniston Kulkosky. Brainwave Neurofeedback Therapeutic Protocol: The Future Psychotherapy for Alcoholism/PTSD/ Behavioural Medicine. 1998.

23. Scott WC, Kaiser D, Othmer S, et al. Effects of an EEG biofeedback protocol on a mixed substance abusing population. Am J Drug Alcohol Abuse. 2005;31(3):455-469.

24. Siegfried Othmer, Sue Othmer. Intra-low frequency neurofeedback for optimum performance. Biofeedback. 2016;44(2):81-89.

25. Siegfried Othmer, Susan F Othmer. Development of the Othmer Method: 1987 to 2016.2016

26. Menon V. Large-scale brain networks and psychopathology: A unifying triple network model. Trends Cogn Sci. 2011;15(10):483-506.

27. Ros T, Munneke MAM, Ruge D, et al. Endogenous control of waking brain rhythms induces neuroplasticity in humans. Eur $J$ Neurosci. 2010;31:770-778.

28. Peniston EG, Kulkosky PJ. Alcoholic personality and alpha-theta brainwave training. Medical Psychotherapy: An International Journal. $1990 ; 3: 37-55$

29. Hanno W Kirk. Restoring the brain: Neurofeedback as an integrative approach to health. UK: Taylor and Francis, CRC Press; 2015. 320 p.

30. Zonta M, Angulo MC, Gobbo S, et al. Neuron-to-astrocyte signaling is central to the dynamic control of brain microcirculation. Nat Neurosci. 2003;6:43-50.

31. Metea M, Newman EA. Glial cells dilate and constrict blood vessels: A mechanism of neurovascular coupling. J Neurosci. 2006;26(11):28622870 .

32. Gordon Gr, Mulligan SJ, MacVicar BA. Astrocyte control of the cerebrovasculature. Glia. 2007;55(12):1214-1221.

33. Parpura V, Basarsky TA, Fang L, et al. Glutamate-mediated astrocyteneuron signaling. Nature. 1994;369:744-747.

34. Bezzi P, Carmignoto G, Pasti L, et al. Prostaglandins stimulate calciumdependent glutamate release in astrocytes. Nature. 1998;391(6664):281285.

35. Schummers J, Yu H, Sur M. Tuned responses of astrocytes and their influence on hemodynamic signals in the visual cortex. Science. 2008;320:1638-1643. 
36. Sasaki T, Kuga N, Namiki S, et al. Locally synchronized astrocytes. Cere Cortex. 2011;21:1889-1900.

37. Poskanzer KE, Yuste R. Astrocytic regulation of cortical UP states. Proc Natl Acad Sci USA. 2011;108(45):18453-18458.

38. Sofroniew MV, Vinters HV. Astrocytes: biology and pathology. Acta Neuropathol. 2010;119(1):7-35.

39. Peters A, Palay SL, Webster HD. The fine structure of the nervous system. 3rd ed. New York: Oxford University Press; 1991. 532 p.

40. Phelps $\mathrm{CH}$. Barbiturate-induced glycogen accumulation in brain. An electron microscopic study. Brain Res. 1972;39(1):225-234.

41. Brown AM, Ransom BR. Astrocyte glycogen and brain energy metabolism. Glia. 2007;55(12):1263-1271.

42. Brown AM, Baltan Tekkok S, Ransom BR. Energy transfer from astrocytes to axons: the role of CNS glycogen. Neurochem Int. 2004;45(4):529536.

43. Pellerin L, Bouzier-Sore AK, Aubert A, et al. Activity-dependent regulation of energy metabolism by astrocytes: an update. Glia. 2007;55(12):1251-1262.

44. Voutsinos-Porche B, Bonvento G, Tanaka K, et al. Glial glutamate transporters mediate a functional metabolic crosstalk between neurons and astrocytes in the mouse developing cortex. Neuron. 2003;37(2):275-286.
45. Garwood CJ, Pooler AM, Atherton J, et al. Astrocytes are important mediators of Abeta-induced neurotoxicity and tau phosphorylation in primary culture. Cell Death Dis. 2011;2(6):e167.

46. M Fotuhi, B Lubinski, M Trullinger, et al. A Personalized 12-week Brain Fitness Program for Improving Cognitive Function and Increasing the Volume of Hippocampus in Elderly with Mild Cognitive Impairment. JPAD. 2016;3(3):133-137

47. Kouijzer ME, De Moor JM, Gerrits BJ, et al. Neurofeedback improves executive functioning in children with autism spectrum disorders. Research in Autism Spectrum Disorders. 2009;3(1):145-162.

48. Escolano C, Aguilar M, Minguez J. EEG-based upper alpha neurofeedback training improves working memory performance. Conf Proc IEEE Eng Med Biol Soc. 2011;2011:2327-2330.

49. Kiluk BD, Carroll KM. New developments in behavioral treatments for substance use disorders. Curr psychiatry Rep. 2013;15(12):1-9.

50. Sinha R. The role of stress in addiction relapse. Current psychiatry reports. 2007;9(5):388-395. 